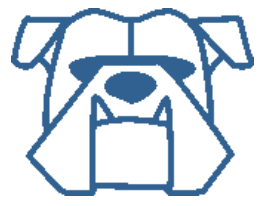

Kettering University

Digital Commons @ Kettering University

$11-28-2011$

Dynamics and Stability of Two-potential Flows in the Porous

Media

Bojan Markicevic

Branko Bijeljic

Homayun K. Navaz

Follow this and additional works at: https://digitalcommons.kettering.edu/mech_eng_facultypubs

Part of the Mechanical Engineering Commons 


\title{
Dynamics and stability of two-potential flows in the porous media
}

\author{
B. Markicevic, ${ }^{1, *}$ B. Bijeljic, ${ }^{2}$ and H. K. Navaz ${ }^{1}$ \\ ${ }^{1}$ Department of Mechanical Engineering, Kettering University, Flint, Michigan, USA \\ ${ }^{2}$ Department of Earth Science and Engineering, Imperial College, London, SW7 2BP, United Kingdom
}

(Received 29 June 2011; revised manuscript received 18 October 2011; published 28 November 2011)

\begin{abstract}
The experimental and numerical results of the capillary-force-driven climb of wetting liquid in porous media, which is opposed by the gravity force, are analyzed with respect to the emergence of a multiphase flow front and flow stability of the climbing liquid. Two dynamic characteristics are used: (i) the multiphase flow front thickness as a function of time, and (ii) the capillary number as a function of Bond number, where both numbers are calculated from the harmonic average of pores radii. Throughout the climb, the influence of capillary, gravity, and viscous force variations on the flow behavior is investigated for different porous media. For a specific porous medium, a unique flow front power law function of time is observed for the capillary flow climbs with or without gravity force. Distinct dynamic flow front power law functions are found for different porous media. However, for capillary climb in different porous media, one is able to predict a unique behavior for the wetting height (the interface between wetted and dry regions of porous medium) using the capillary and Bond number. It is found that these two numbers correlate as a unique exponential function, even for porous media whose permeabilities vary for two orders of magnitude. For climbs without the gravity force (capillary spreads), the initial climb dynamics follows this exponential law, but for later flow times and when a significant flow front is developed, one observes a constant value of the capillary number. Using this approach to describe the capillary climb, only the capillary versus Bond number correlation is needed, which is completely measureable from the experiments.
\end{abstract}

DOI: 10.1103/PhysRevE.84.056324

PACS number(s): 47.56.+r, 47.55.nb, 47.61.Jd

\section{INTRODUCTION}

The displacement flows in porous media are free boundary flow problems in which an originally present fluid is forced out by a displacing fluid entering the porous medium from its boundary(ies). Capillary, gravity, and viscous forces govern the dynamics of the displacement flow, where the forces can act in arbitrary directions, influencing the form and stability of the free flow interface. The magnitude and relative importance of these forces vary with the length scale studied. Soil moisture transport, underground water flow, and oil recovery are flow examples on the kilometer scales (e.g., [1,2]). The meter to millimeter scales are pertinent to the engineering applications in textile engineering, paper coating, flow in fibrous filters, and drying [3-6]. Further reduction in scales is found in more recent applications, ranging from the fuel cells and microfluidic devices to the carbon nanotube and its fluidic arrays $[7,8]$. However, most often, capillary flows or capillary force opposed by gravity-driven displacement (two-potential) flows are met in practice. Due to the porous medium heterogeneity, the momentum dissipation and later multiphase flow front development have a large influence on the flow dynamics. Further disturbances in the flow interface may be caused by nonlinear effects such as inertia or fluid/solid contact angle dependence on the fluid velocity. The dynamics and stability of the two-potential flows in porous media are complex phenomena that have not been fully understood.

For the capillary (or external) force-driven displacement flows in homogeneous media it has been shown analytically [9] that the dynamics of the advancing fluid front with the interface between two phases sharply defined follows the square root

*bmarkice@kettering.edu dependence on time. Actual porous media are heterogeneous materials with a broad range of pore sizes and, as a result of that, both sharp interface and flow front (an interface of finite thickness) dynamics can be observed. The interplay of the displacement rate, medium heterogeneity, and forces present cause, in some cases, more complex flow to emerge, where the flow front is formed rather than the sharp interface. In either physical case, the sharp interface position [10-12] or flow front position and thickness [13] in time depend on the locally random permeability field, which is often quantified with the variance of its logarithmic values. Once the capillary climb is opposed by gravity force, the maximum climbing height exists for both sharp interface [14-16] and flow front behavior. When a multiphase flow front of displacing fluid exists, the Richards equation can be used to model the dynamic saturation gradients within the flow front and to predict the climb dynamics, as shown by Lockington and Parlange [17]. The flow parameters within the flow front, namely, capillary pressure and relative permeability, can be measured experimentally [18] or from a matching method in which the numerical solution is used to deduce flow parameters after some experimental and numerical flow characteristics are matched [19,20]. The presence of a flow front has been observed in experiments on the capillary rise of water through a packing of glass beads by Delker et al. [21] and Lago and Araujo [22], where the climbing height is a two-power function of time, with the power at early times being larger compared to the power for longer climbing times. The changes in the climb dynamics are explained in full using the numerical solutions [23], where it is shown that the flow front and multiphase flow emerge as flow becomes slower for larger climb heights.

In the stable displacement flow, the phases in the flow front are not segregated, giving rise to the flow front with both 
phases as continuous domains rather than fingering of one phase into another [24,25]. However, for the flows in which the formation of large-scale clusters is preferred due to the magnitude of the gravity, viscous, or capillary forces, fingering can be observed [26,27]. It has been shown that dependent on the viscosity ratio of the two phases and the capillary number, the viscous and capillary fingers can be developed [28]. The flow stability is further influenced by additional gradients such as gravity or thermal gradients, where capillary and gravity length scales are proportional to the capillary and Bond number [29-31]. Thus, for liquid displacing gas phase, except for fast or gravity-stabilized flows as quantified using the generalized Bond number [29,32], the multiphase flows produce an irregular, unstable interface with dynamic changes in the distribution of fluid phases. With respect to the emergence of a multiphase flow front and stability for the capillary climb, it has been shown [23] that the capillary climb is initially single-phase displacement flow, followed by development of a stable (without fingering) flow front, while some capillary fingering may be observed closer to the climb cessation, in which the formation of clusters may be preferred due to the slow flow. Still, the cluster size is limited due to the flow stabilization by gravity force which counteracts the capillary-induced instability.

We study dynamic behavior and the stability of twopotential capillary climb flows into different porous media. Using a dynamic capillary network model [33] and its comparison with the experimental results for the capillary climb [23] we investigate the climbing dynamics in detail, where both the flow front thickness and wetting height are used as characteristic properties. We show how different combinations of forces influence the climb dynamics. Moreover, we present how the large variations in forces acting on the liquids can be normalized using the capillary and Bond number, where we use the experimental results of Lago and Araujo [22]. Using the interfacial front position and the velocity of front propagation for glass bead packs and Berea sandstone from the experiments, together with the numerically predicted average pore radius and analytically predicted permeability, a unique master curve in the exponential form construes all results. We demonstrate that for the climbs without the gravity force, the initial climb dynamics follows this exponential law, but for later flow times and when a significant flow front is developed, a constant value of the capillary number is observed.

\section{PROBLEM FORMULATION}

In the schematic in Fig. 1, the capillary climb problem is depicted. Under the influence of the capillary force, a wetting liquid is imbibed by a porous medium from the bottom boundary with the liquid flow opposed by gravity force given with symbol $g$. The sum of the capillary and gravity force is equal to the viscous force of the liquid flow, and the viscous force and flow velocity decrease as climbing height and gravity force increase. The flow velocity can be altered further by placing a low permeable layer of thickness $l$ and permeability $K_{l}$ at the bottom of the porous medium bed. For the liquid at rest, the viscous force is equal to zero, stipulating that the capillary climb takes place until the capillary and gravity force equilibrate. Initially the climbing velocity is high, with the
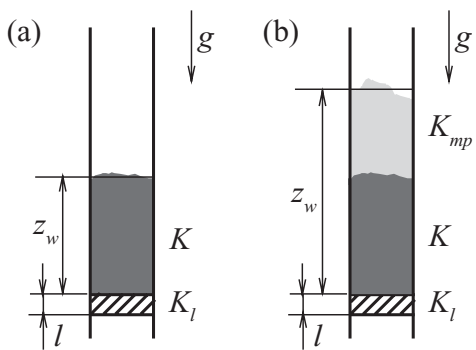

FIG. 1. Capillary climb of wetting liquid into a porous medium opposed by gravity force: (a) initial single-phase displacement flow and (b) multiphase flow front development for larger heights. Liquid enters the medium from the bottom boundary.

interface between the wetted and dry portion of the porous medium well defined (sharp interface) and the wetted portion of the porous medium fully saturated. The liquid climb is defined as a single-phase displacement flow, as shown with a dark gray region, and climbing height $z_{w}$ in Fig. 1(a). As the climb progresses, the multiphase flow front of finite thickness can develop, as shown in Fig. 1(b) with a light gray region, where the climbing height $\left(z_{w}\right)$ is again defined as an interface location between the wetted and dry regions of the porous medium.

It is obvious that the dynamics of the flows given in Figs. 1(a) and 1(b) differ, where for single-phase displacement flow, the flow resistance is related to the porous medium single-phase flow permeability $(K)$ and the capillary pressure is proportional to the averaged capillary pressure of the overall medium. On the other hand, once the multiphase flow front develops, the flow resistance of the multiphase region can be related to the multiphase flow permeability $\left(K_{m p}\right)$ and the capillary pressure is an average of local capillary pressures of the local points preferable for the liquid to climb. The differences in the local flows at the interface stem from the fact that the porous media are heterogeneous materials consisting of the pores (voids) of different sizes. The pores exhibit different flow resistances and capillary pressures. From the potential condition for spontaneous flow, for the capillary climb to take place in an interfacial pore $(i)$ of radius $r_{p, i}$ and having the height $z_{w, i}$, the liquid phase potential $\left(\varphi_{\text {int }, i}\right)$ has to be negative. The local potential in an arbitrary pore $(i)$ consists of capillary and gravity contributions:

$$
\varphi_{\mathrm{int}, i}=-\frac{2 \sigma}{r_{p, i}} \cos (\theta)-\rho g z_{w, i}
$$

where $\sigma$ and $\rho$ are liquid surface tension and density, $g=$ $-9.81 \mathrm{~m} / \mathrm{s}^{2}$, and $\theta$ is a solid/liquid contact angle.

To solve the flow problem which accounts for the local flows at the interface, the capillary network model with the dynamic boundary condition is used. Full details of the model with descriptions of the subtle multiphase flow physics can be found in Markicevic and Navaz [13] and Bijeljic et al. [23]. In the model the porous medium is replaced by a network consisting of the volume elements, pores, and the flow resistive elements, throats. For each pore at the interface, the liquid potential is defined using Eq. (1) and the flow rate into the interfacial pore is calculated from the known pressures in all pores of the liquid phase. The pressure is calculated from the conservation equations (mass and momentum), and therefore 
the influence of viscous, capillary, and gravity forces on the liquid distribution is accounted for. From such calculated pressure the local flow at the interface is fully solved, where for each pore at the interface the flow rate can be positive or negative depending on the local force balance. However, in the next step, the flow direction in each of pores can be reverted. Having flow rates defined, the local piston flow and snap-off are determined where the sum of all local flows gives the measure of the flow dissipation at the interface. Throughout time, the fluid flows along its preferential paths, where locally the liquid phase can protrude further into the dry part of the porous medium or gas phase can be entrapped by liquid phase, forming liquid ganglia and gas clusters, respectively. The sizes of both clusters and ganglia are a measure of the flow instability.

In Eq. (1) a local condition for the climb to take place is defined. After averaging for the liquid climbing the porous medium bed, the force balance at the wetting interface requires that the viscous force is equal to the sum of the capillary and gravity forces. The viscous force depends on both climbing height $\left(z_{w}\right)$ and velocity $(u)$. Expressing the force balance per unit area, one can write

$$
u \mu \frac{z_{w}}{K}=\frac{2 \sigma}{r_{p, h}} \cos (\theta)+\rho g z_{w},
$$

where $\mu$ is liquid viscosity. In Eq. (2) the single-phase flow permeability $(K)$ and harmonic average of pore radii $\left(r_{p, h}\right)$ are used, where $K$ and $r_{p, h}$ can be used as long as the climb can be described as a single-phase displacement flow; the condition is satisfied for all pores having high negative potential. Throughout the climb, the magnitudes of gravity and viscous forces change, and so do the relative ratios of viscous and gravity force to the capillary force. These two ratios are referred to as the capillary $(\mathrm{Ca})$ and the Bond (Bo) numbers and they are equal to

$$
\mathrm{Ca}=\frac{u \mu}{2 \sigma \cos (\theta)} \frac{z_{w} r_{p, h}}{K} \quad \text { and } \quad \mathrm{Bo}=\frac{\rho g z_{w} r_{p, h}}{2 \sigma \cos (\theta)},
$$

where $K$ can be calculated using the Kozeny equation and $r_{p, h}$ is easy to determine from a known pore size distribution of the porous medium (note that superficial velocity of the wetting interface is found from $u=\phi d z_{w} / d t$ ). The interface climbing velocity $(u)$ can be altered by adding a low permeable layer at the bottom of the porous column (layer of $l$ and $K_{l}$ in Fig. 1), where its presence can be easily accounted for by defining an effective permeability $K_{\text {eff }}$ that is influenced by $K_{l}$ and $K$ as follows: $\left(l+z_{w}\right) / K_{\text {eff }}=l / K_{l}+z_{w} / K$ (note that for $l=$ $\left.0, K_{\text {eff }}=K\right)$. In this case the capillary number in Eq. (3) is calculated using $K_{\text {eff }}$ instead of $K$. Finally, $u$ can be altered further by changing the gravity or even setting $g=0$, for which the capillary climb reverts to the capillary spread problem [34] and $\mathrm{Bo}=0$.

Regardless of the forces present ( $l$ and/or $g$ ), the capillary climb decelerates, and for sufficiently small velocity (capillary number), the multiphase flow front emerges as a result of the liquid climbing the porous medium along preferential flow paths for which the interfacial pores potential is negative. The sharp wetting interface transforms into the flow front of finite thickness, and as capillary number decreases, the flow front thickness grows. For the gravity force present, the climb ceases once the potential at the interface is equal to zero, whereas for capillary spreads ( $g=0)$, the spread should come to the end due to the sufficiently large momentum dissipation within the flow front. The flow front extends from (i) the interface which separates fully and partially saturated regions of porous medium, and to (ii) the interface between wetted and dry fraction of the overall domain. The latter interface is referred to as the wetting interface. Equation (2) describes again the force balance at the wetting interface, where effective pore radius $\left(r_{p, e f}\right)$ and effective permeability $\left(K_{\text {eff }}\right)$ have to be used. The value of $r_{p, e f}$ is determined as a harmonic average of pore radii for pores preferential for flow to take place, and $K_{\text {eff }}$ is found from the low permeable layer, fully saturated region, flow front multiphase permeabilities, $\left(K_{l}, K, K_{m p}\right)$ and each region thickness. Finally, the capillary and Bond number, $\mathrm{Ca}$ and Bo, are found using $r_{p, e f}$ and $K_{\text {eff }}$ in Eq. (3) and the wetting interface superficial velocity, $u=\phi d z_{w} / d t$. (Note that there is an overestimation of $u$ as the liquid saturation is smaller than one.)

\section{RESULTS AND DISCUSSION}

The first dynamic characteristic we study numerically is the climbing height as a function of time $z_{w}(t)$. The computed results for four combinations of $(g, l)$ are given in Fig. 2. In the numerical solution, the porous medium is represented as a regular cubic network of pores connected by throats, with both pores and throats having uniform distribution [23]. The threshold potential condition is used at the free interface for liquid climb. The numerical solution predicts $z_{w}(t)$ curves in both single- and multiphase flow front regions, where $g<0$ and porous medium local heterogeneities produce two different slopes along the $z_{w}(t)$ curve. Note that $l>0$ causes the slope of $z_{w}(t)$ to increase. Delker et al. [21] and Lago and Araujo [22] have studied capillary climb experimentally by measuring the climbing height as a function of time. For the $l>0$ and $g \neq 0$ flow case, they observed flow dynamics characterized by two distinct flow mechanisms: the climb starts as a single-phase displacement flow which is then followed by development

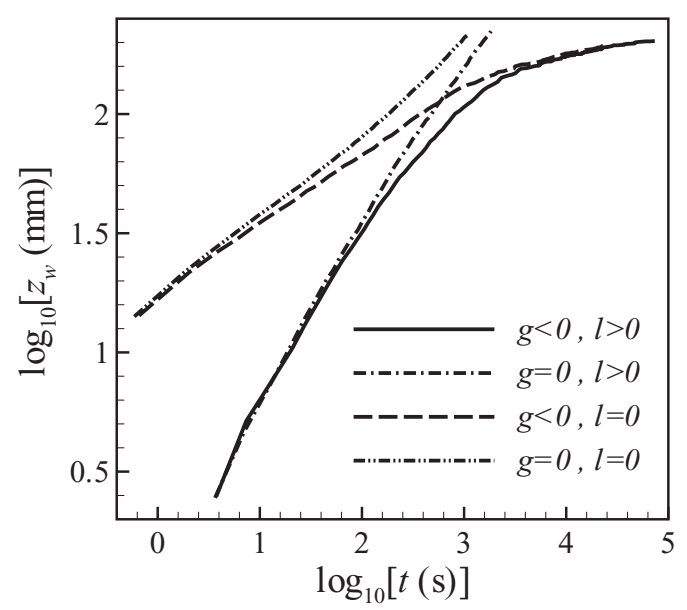

FIG. 2. Numerical solution of the climb dynamics given as a climbing height $\left(z_{w}\right)$ as a function of time $(t)$ for the low permeable layer $(l)$ and gravity $(g)$ equal to or different than zero. Single-power $(g=0)$ or two-power $(g<0)$ dynamics is observed. The value of the power for small climb heights is different for $l=0$ and $l>0$. 
of a multiphase flow front for larger climbing heights. The transition from a single- to multiphase flow pattern can be clearly observed from the change of the slope in the logarithmic axes plot of height as function of time $z_{w}(t)$. Using capillary network models, Bijeljic et al. [23] have solved numerically the same cases as in the experimental study of Lago and Araujo [22], obtaining a very good agreement between experimental and numerical $z_{w}(t)$ curves and slopes calculated from $z_{w}(t)$ logarithmic plots. In the single-phase displacement flow in a homogeneous porous medium of permeability $K$, the slope should be equal to one half as calculated from the Washburn equation. Placing a low permeable layer $\left(K_{l}, l\right)$ at the column bottom, the slope increases due to the larger flow resistance which is quantified by defining the effective permeability $\left(K_{\text {eff }}\right),\left(l+z_{w}\right) / K_{\text {eff }}=l / K_{l}+z_{w} / K$. Due to the gravity, the maximum climbing height can be determined in homogeneous porous media. The summary of $z_{w}(t)$ analytical solutions for four distinct combinations ( $l$ and $g$ equal to and/or different than zero) are given in Bijeljic et al. [23], where clearly, the Washburn equation fails to predict $z_{w}(t)$ in the multiphase flow front region. (It does not allow for the existence of the multiphase flow.)

The experimental measurements of water climbing the glass bead beds are carried out for the $(g<0, l>0)$ case, and therefore the $z_{w}(t)$ curve has two slopes. As described in Delker et al. [21], smooth wetted front advancement is observed initially, while later for larger climbing heights, more like hopping flow is observed. The liquid from one point of porous medium is carried further, opening the originating point, which is in the next step filled by liquid from the domain inlet. These two steps alternate, giving a notion that liquid hops between points. As shown in Fig. 3, our numerical solution supports such a mechanism, where in the single-phase flow the wetted interface climbs the porous medium. Once the wetted interface reaches the points of minimum threshold potential, a partially saturated region starts to grow with its lower bound remaining stationary. Hence, the width of the partially wetted region depends on the porous medium heterogeneity, where the flow in the partially wetted region is maintained by flow between the liquid inlet boundary (bottom boundary in Fig. 3) and the highest fully wetted region of the porous medium [around $z=120 \mathrm{~mm}$ in Figs. 3(b) and 3(c)]. The liquid flows (hops) into the partially wetted region from the highest fully wetted region due to the difference of capillary pressures between the small and large pores. The wetting interface at the top of the flow front (between the wet and dry part of the porous medium) remains essentially smooth, except for the small fingers, as can be seen in Fig. 3(c), showing that the flow is stable. Finally, the flow stability can be caused by the gravity force direction and by the domain size which restrict the emergence of large clusters and flow instability.

The numerical results in Fig. 2 suggest that there is a difference in the capillary numbers for all four $(g, l)$ flow cases. The capillary flow which is not opposed by gravity and without the low permeable layer at the column bottom $(g=0, l=0)$ is fastest, and the flow resembles the single-phase displacement flow. However, as liquid progresses into porous medium, the capillary number decreases and a smooth interface transforms into a flow front with a partially wetted porous medium. By adding a low permeable layer $(g=0, l>0)$, the liquid velocity and capillary number decrease and the flow front emerges for a smaller wetting height. On the other hand, the gravity $(g<0$, $l=0$ ) alters the flow front thickness at the later times, whereas initial flow resembles the single-phase flow as capillary force is higher compared to the gravity force. In order to slow down the flow for small climbing height and obtain the multiphase flow, a low permeable layer needs to be added $(g<0$, $l>0)$. All these changes are summarized in Fig. 4, where

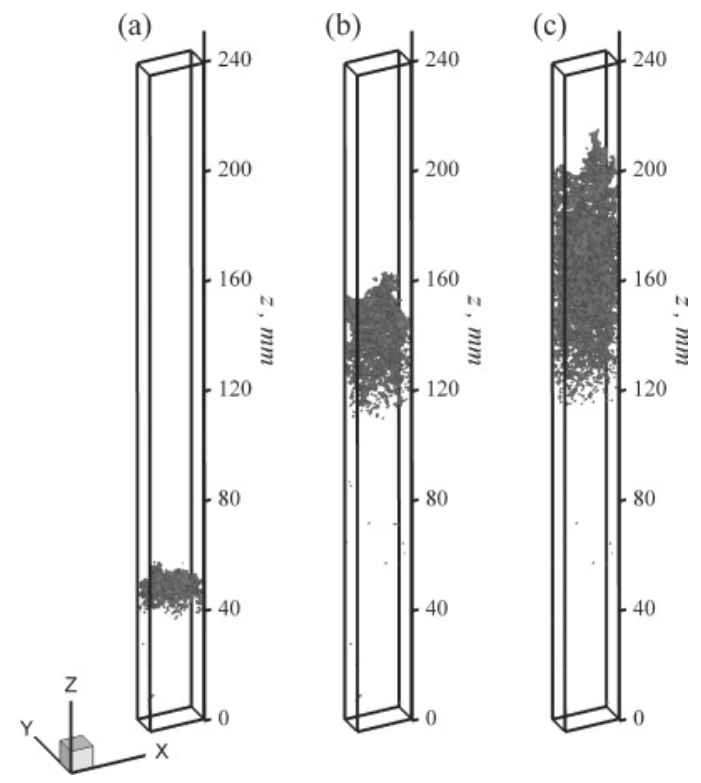

FIG. 3. Flow front development given as a saturation iso-surface ( $s=0.95$ ) in time for capillary climb, $g<0$ and $l>0$, where after reaching the potential lower limit, the interface between fully and partially wetted regions remains stationary $\left(z_{w} \approx 120 \mathrm{~mm}\right)$.

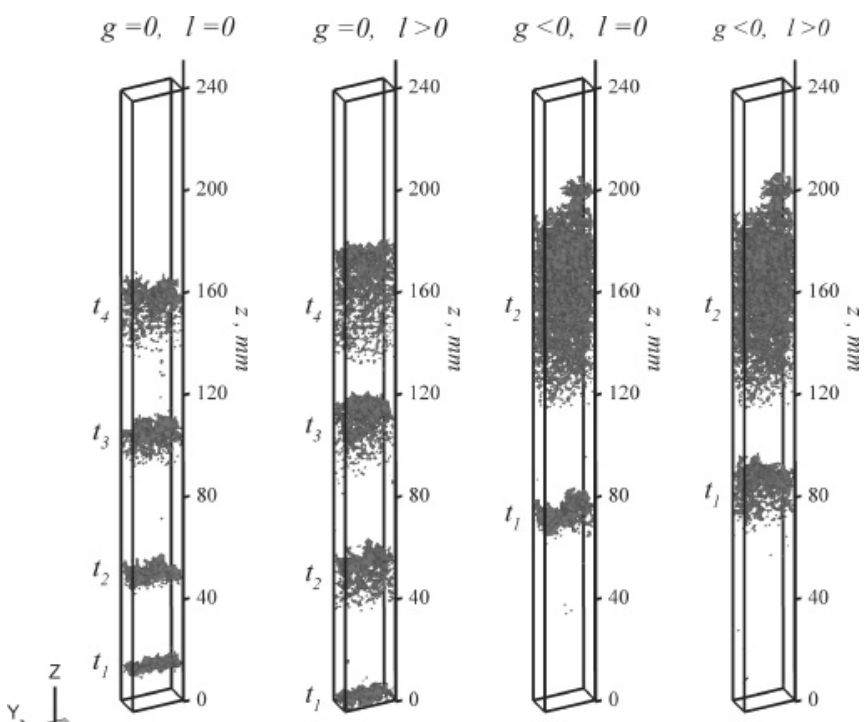

(b)

(c)

(d)

FIG. 4. Flow front development for four cases of $(g, l)$ investigated, where the flow front becomes thicker in time as the capillary number decreases. For longer times and $g<0$, the same flow front thickness is found (c and d). (Note that times $t_{1}-t_{4}$ are different in each case a-d.) 


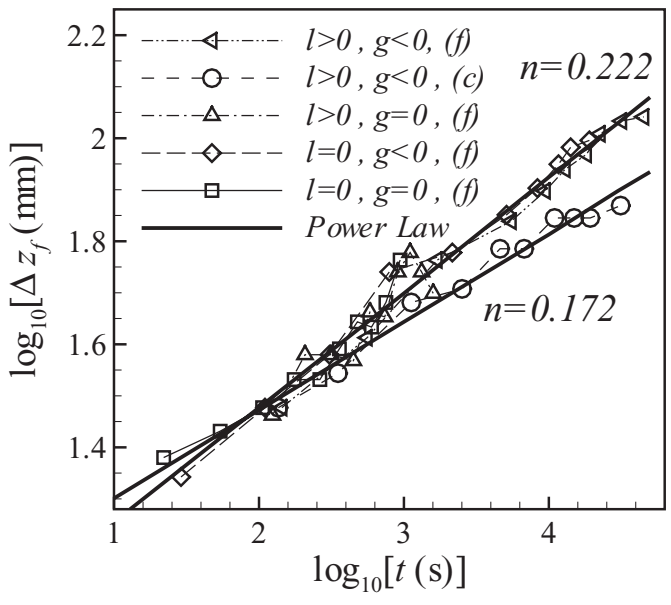

FIG. 5. Power law function of flow front thickness versus time, where the same power $(n)$ is calculated for a specific porous medium irrespective of the forces present $(g, l)$. The power is the porous medium specific (fine and coarse glass beads).

all four cases are depicted. The flow front thickness and how it changes in time is visualized by plotting the iso-surfaces with the liquid saturation $s=0.95$. For the two cases when $g<0$, the flow front thickness just before the climb ceases is not affected by presence of the low permeable layer $(l)$, as observed in Figs. 4(c) and 4(d). In both cases the flow is slow, and before a complete stop, the liquid reaches its equilibrium distribution (from the equilibrium of gravity and capillary force). As already shown in Fig. 3, limited fingering is observed for flow cases with gravity, whereas for flows governed by capillary force only, almost no fingers are observed. Hence, all four flow cases are stable where the single-phase displacement flow transitions to the flow with the multiphase flow front.

In Delker et al. [21] and Lago and Araujo [22], there are no reported results of how the flow front thickness changes as a function of time. However, having the experimental results and numerical results from Bijeljic et al. [23] for climbing height agree for $(g<0, l>0)$, the flow front thickness $\left(\Delta z_{f}\right)$ is determined from the numerical results, where the axial saturation profiles $s(z)$ are calculated from the time solutions given in Fig. 4 (see Bijeljic et al. [23]) for all four combinations of $(g, l)$. As shown in Fig. 5, irrespective of values $(g, l)$ and for a specific porous medium, i.e., glass beads, $d_{p}=165 \mu \mathrm{m}$ (referred to as fine-letter $f$ ), and $\Delta z_{f}$ is a unique function of time $(t)$ and can be expressed as a power law with the power $n=0.222$. This suggests that the multiphase flow front development always follows the same pattern and that it emerges earlier or later in time due to different force balance. On the other hand, changing the porous medium, i.e., glass beads, $d_{p}=275 \mu \mathrm{m}$ (referred to as coarse-letter $c$ ), produces a flow front of different structure and $\Delta z_{f}$ grows in time in a different way. Thus, one observes a different power, $n=0.172$, compared to the previous sample. Finally, using the capillary number $(\mathrm{Ca})$ instead of time does not give any conclusive result with $\Delta z_{f}$ versus Ca scatter for the same $d_{p}$ and the four $(g, l)$ combinations.

From Eq. (3) the capillary number is a function of the product of climbing height and velocity $\left(z_{w} u\right)$, whereas the Bond number is a function of climbing height $\left(z_{w}\right)$ only. For glass bead beds of $d_{p}=165 \mu \mathrm{m}$ and $d_{p}=275 \mu \mathrm{m}$, the
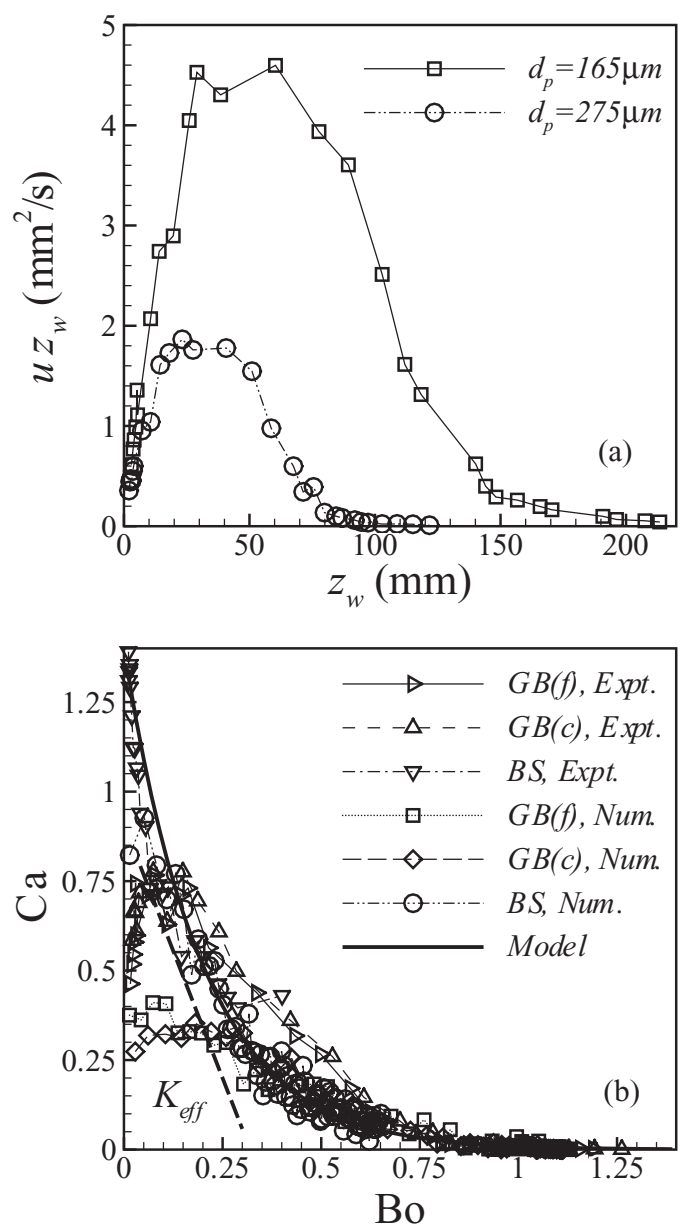

FIG. 6. Capillary and Bond number correlation of (a) pertinent values $u z_{w}$ and $z_{w}$ for two experimental measurements and (b) reduction using the medium effective permeability and harmonic average of pore radii for two glass bead (GB) beds and Berea sandstone (BS). The maximum $\mathrm{Ca}$ is observed due to the changes in the effective permeability.

experimental values of $z_{w} u$ and $z_{w}$ are correlated, showing two distinct behaviors as depicted in Fig. 6(a). The value of maximum $z_{w} u$ is higher and shifted to larger $z_{w}$ as $d_{p}$ decreases, which implies that the capillary flows are faster in fine porous media and that the increase in the driving force from the capillary pressure is greater than the decrease in the permeability (overall $p_{c} K$ product increases as $d_{p}$ decreases). The maximum in $z_{w} u$ is due to the influence of a low permeable layer $\left(l, K_{l}\right)$, where effective permeability $\left(K_{\text {eff }}\right)$ increases as climbing height $\left(z_{w}\right)$ increases, $\left(l+z_{w}\right) / K_{\text {eff }}=$ $l / K_{l}+z_{w} / K$, and so does $z_{w} u$. Once $z_{w}$ is sufficiently large and $K_{\text {eff }}$ is almost identical to $K$, the product $z_{w} u$ starts to decrease due to gravity. (Note that without gravity the product $z_{w} u$ is a constant, as stipulated from Darcy's law.) However, instead of correlating $z_{w} u$ and $z_{w}$, it is of interest to reduce the data onto Bo and Ca dependence as shown in Fig. 6(b). In order to calculate the capillary and Bond number, the average pore radius needs to be known, and in Fig. 6(b) the pore harmonic average radius $\left(r_{p, h}\right)$ is used. Using the values of pore radii as a uniform distribution from Bijeljic et al. [23] and effective permeability from $\left(l+z_{w}\right) / K_{\text {eff }}=l / K_{l}+z_{w} / K$, 
the experimental and numerical results for glass beads $d_{p}=$ $165 \mu \mathrm{m}$ and $d_{p}=275 \mu \mathrm{m}$ [referred to as fine $(f)$ and course (c) samples] and Berea sandstone are collapsed to a single curve. In Fig. 6(b) there is a deviation from the single curve dependence for glass beads and small climbing height, where again a capillary number maximum is observed. The maximum is around $\mathrm{Ca}=0.75$ and 0.35 for $d_{p}=165$ and $275 \mu \mathrm{m}$, respectively, whereas no such maximum is found for Berea sandstone. Keeping in mind that the low permeable layer $\left(l, K_{l}\right)$ is used only for the glass bead beds, the results suggest that $K_{\text {eff }}$ correction is not entirely adequate to capture the climb for low heights and that the usage of $K_{\text {eff }}$ in calculating the capillary number from Eq. (3) is not sufficient to account for the overall physics of the climb. This is evidenced from the Berea sandstone data, where for $\left(l, K_{l}\right)$ absent, the $\mathrm{Ca} \sim$ Bo curve falls on top of the glass bead data. Further analysis of results in Fig. 6(b) reveals that both $\mathrm{Ca}$ and Bo numbers take values up to 1.25 , implying that (i) viscous force is greater than the capillary force (here the gravity is negligible from small climbing height) and (ii) gravity force is greater than the capillary force (here the viscous force is negligible as liquid is almost brought to a complete stop). Both physical discrepancies, described by both $\mathrm{Ca}$ and Bo greater than one, are due to the porous medium heterogeneities, where smaller pores (and high capillary pressures) cause larger climbing height and liquid velocities compared to their counterparts calculated for the homogeneous sample (macroscopically averaged).

As opposed to the Washburn equation, in the model development of capillary climb which accounts for the presence of the multiphase flow front, Lockington and Parlange [17] have used exponential function for relative permeability and capillary pressure as a function of saturation. Still, an additional parameter $(A)$ is needed in their model. As shown in Fig. 6(b), except for the influence of $K_{\text {eff }}$ for small climbing height, both glass bead beds and Berea sandstone show a single dependence $\mathrm{Ca}$ as a function of Bo which follows an exponential decay shown with the solid line. Hence, one can write

$$
\mathrm{Ca}=b \exp (-a \mathrm{Bo}),
$$

where $a$ and $b$ are model parameters and have the same value for glass beads of $d_{p}=165$ and $275 \mu \mathrm{m}$ and Berea sandstone. Figure 7 shows a full comparison for these three porous media, where the results are presented in linear and logarithmic axes. Since the maximum of the capillary number is found for very small climbing height, the points before a $\mathrm{Ca}$ maximum is reached are excluded from the comparison in Fig. 7. The experimental results (different triangles) are slightly above the numerical predictions (other symbols), and there is a very good comparison against exponential function from Eq. (4) (thick solid line). From the results shown in the logarithmic plot, the behavior of the experimental and numerical results for Bo close to one reveal that once $\mathrm{Ca}<0.01$, the Bond number essentially does not change [seen as almost a vertical trend of Bo values, right bottom corner in Fig. 7(b)]. Finally, the values of parameters $a$ and $b$ are determined from all data to be equal to $a=4.96$ and $b=1.39$.

The capillary climb results show that there are two characteristic heights, where the first one is the height for which a maximum value of the capillary number is found
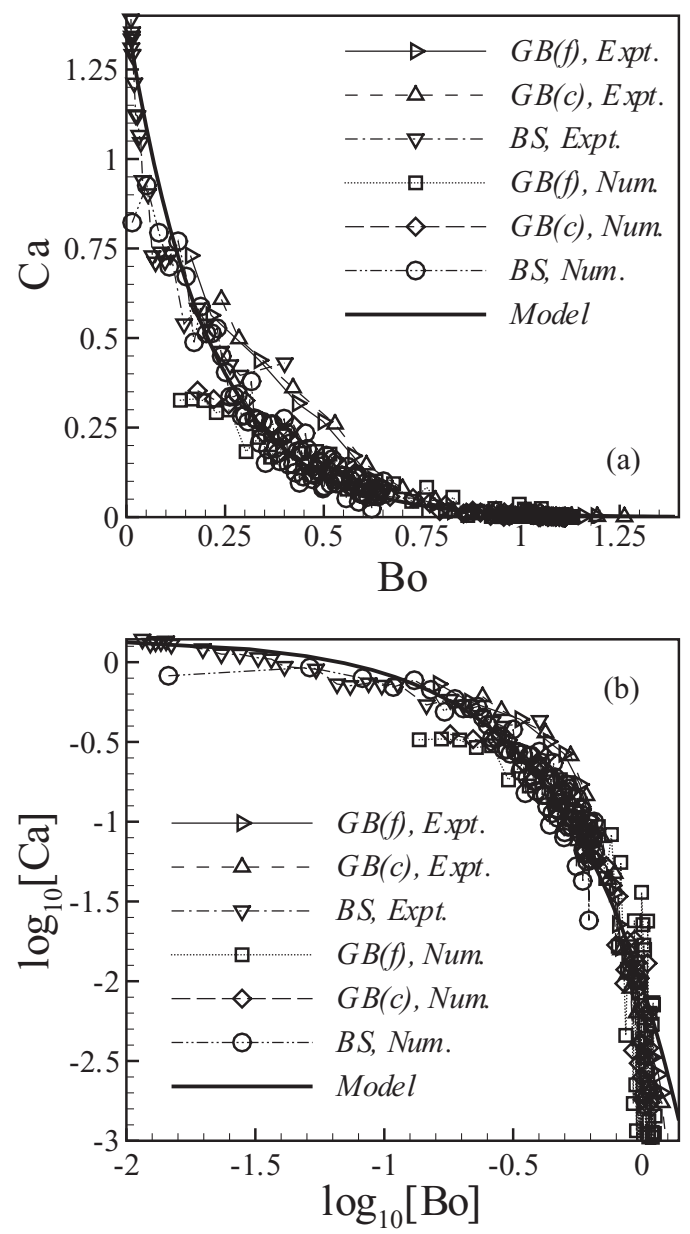

FIG. 7. Comparison of experimental and numerical reduced data with the exponential correlation of capillary and Bond number, $\mathrm{Ca}=b \times \exp (-a \times \mathrm{Bo})$. The data for small climbing height and the corresponding effective permeability $\left(K_{\text {eff }}\right)$ are excluded: (a) linear axes and (b) logarithmic axes.

$\left(z_{m}\right)$ and the second is the height for which a flow front of significant thickness starts to develop $\left(z_{t}\right)$. The normalized climbing height can be defined $\left(\Delta z_{w}\right)$ as

$$
\Delta z_{w}=\frac{z_{w}-z_{m}}{z_{t}-z_{m}},
$$

where for $\Delta z_{w}>1$, the multiphase flow front becomes significant. Clearly, the maximum capillary number is observed only in the case when the low permeable layer at the bottom of the column is present $\left(l, K_{l}\right)$, and otherwise, $z_{m}=0$ is set in Eq. (5). It is of interest if $\Delta z_{w}$ can be correlated with the capillary number, as $\Delta z_{w}>1$ occurs only for very slow flow, $\mathrm{Ca}<0.01$. The climb results are correlated both to the exponential and power function, with both correlations having two parameters $\left(a_{z}, b_{z}\right)$ and $(\alpha, \beta)$, respectively:

$$
\Delta z_{w}=b_{z} \exp \left(-a_{z} \mathrm{Ca}\right) \text { and } \Delta z_{w}=\beta \mathrm{Ca}^{-\alpha} .
$$

Using $\left(z_{m}, z_{t}\right)=(26,140)$ and $\left(z_{m}, z_{t}\right)=(18,80)$ (all in millimeters), both glass beads $d_{p}=165 \mu \mathrm{m}$ and $d_{p}=$ $275 \mu \mathrm{m}$ exhibit a single behavior as shown in Figs. 8 and 9 for exponential and power function, respectively. Surprisingly, in the exponential dependence $\left(a_{z}, b_{z}\right)$ is very similar to the values 

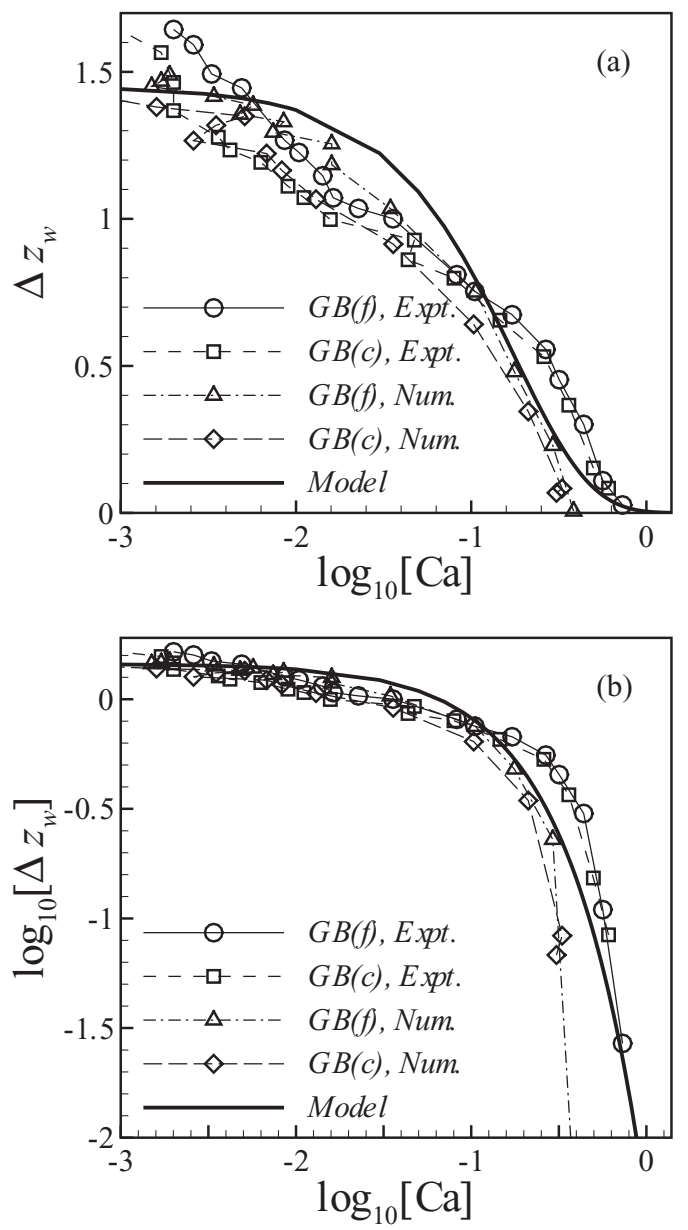

FIG. 8. Dependence of normalized wetting interface $\left(\Delta z_{w}\right)$ on the capillary number $(\mathrm{Ca})$, where $\Delta z_{w}<1$ occurs for lower heights and smaller gravity force, and $\Delta z_{w}>1$ is the climb for prevailing gravity force. The results follow the exponential function (thick line).

of $(a, b)$ from Eq. (4), where $a_{z}=5.67$ and $b_{z}=1.46$. The exponential function (Fig. 8) can be used in the whole range of $z_{w}\left(\Delta z_{w}>0\right)$, whereas the power function (Fig. 9) can be used only to predict the width of the multiphase flow front $\left(\Delta z_{w}>\right.$ 1 ), with the power $\alpha=0.187$. Finally, for $\mathrm{Ca}<0.01$, numerical results show that the capillary climb is close to ceasing, as observed from leveled-off results for $\Delta z_{w}$ as Ca decreases further. Finally, for $\mathrm{Ca}>0.01$, numerical results show a very similar slope $(\alpha=0.187)$ to the experimental results.

In the analysis of the numerical and experimental results for $(g<0, l>0)$, the liquid velocity and the capillary number are calculated from the viscous force that is a result of the capillary and gravity forces. Having the numerical solution validated with the experimental results for $(g<0, l>0)$ [23], the influence of $g$ and $l$ on the climb dynamics is investigated numerically and the results are correlated using the capillary and Bond numbers for glass beads $d_{p}=165 \mu \mathrm{m}$. For two cases in which $g=0$ (capillary spread), the Bond number is still calculated using Eq. (3) using the wetting interface $\left(z_{w}\right)$. Figure 10 summarizes the experimental results (circles) for $(g<0, l>0)$ and numerical results for all four combinations of $(g, l)$. The exponential dependence in Eq. (4) is also given (thick solid line), where the same values of parameters $a$ and
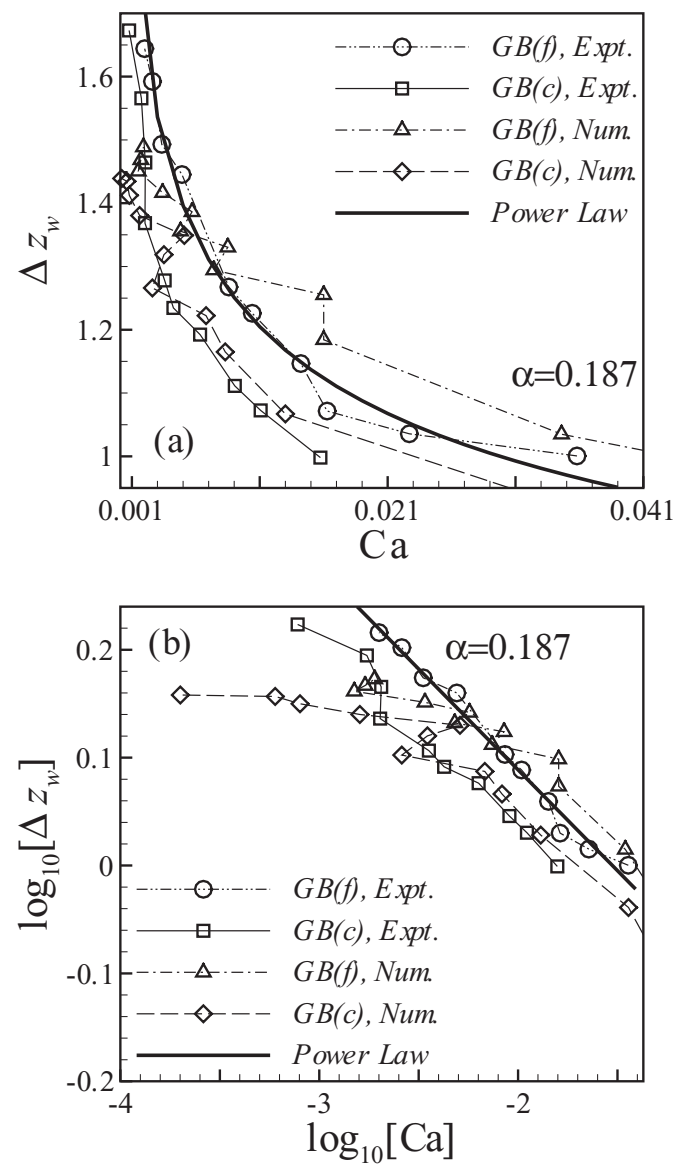

FIG. 9. Power law approximation of $\Delta z_{w}$ as a function of Ca for $\Delta z_{w}>1$. For both experimental and numerical results for distinct media (fine and coarse), the same power $\alpha=0.187$ is calculated. The numerical results for $\Delta z_{w}$ (triangles and rhombuses) approach a constant value implying that the liquid reached close to the maximum climbing height.

$b$ are used as in Fig. 7, $a=4.96$ and $b=1.39$. The flow case with gravity present, $g<0$, and unaltered viscous force, $l=0$, (rhombuses) follows the exponential dependence, and furthermore, no local maximum of $\mathrm{Ca}$ is observed for this case as $K_{\text {eff }}=K$. For the cases without gravity, $g=0$, the capillary number is higher and it becomes constant $(\mathrm{Ca} \approx$ const.) after liquid reaches some distance (herein Bo $\sim 0.4)$. Since for $l=0$ we calculated the capillary number in Eq. (3) using constant $K_{\text {eff }}=K$ and $r_{p, e f}=r_{p, h}$, the wetted front satisfies $z_{w} d z_{w} / d t=$ const., and $z_{w}$ should be again a square root function of time. However, the results of Lago and Araujo [22] for Berea sandstone reveal that the power is lower than one half $(n \approx 0.43)$ due to the momentum dissipation. This is observed for our numerical cases for $g$ $=0$ as small intermittencies of the capillary number. For this flow $(g=0)$, the viscous force and liquid velocity is caused by capillary force only, and therefore, the velocity needs to be reduced for the gravity negative contribution $\left(u_{g}\right)$. Setting $u_{g}=K_{\text {eff }} \rho g / \mu$, we find that $u-u_{g}$ is less than zero for Bo > 0.4 (the region where $\mathrm{Ca}$ is constant) with the multiphase flow front present, but without the capillary instability which has been previously reported for nonwetting liquid [29,32]. Finally, it should be kept in mind that both experimental results 

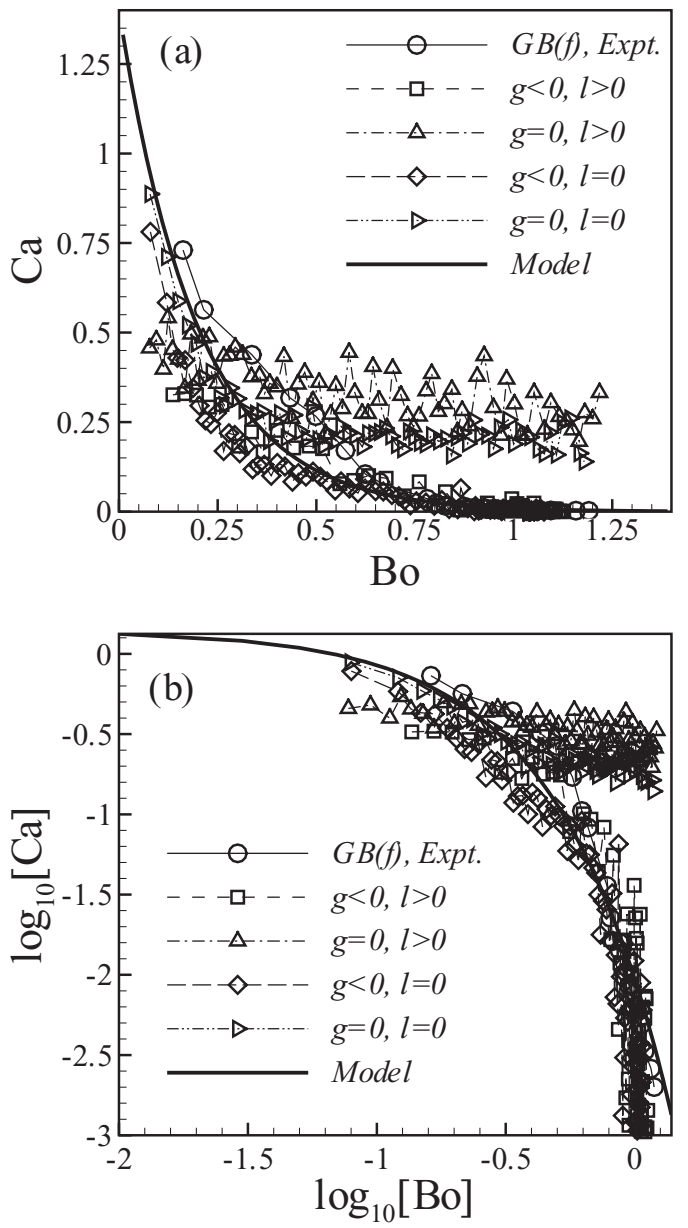

FIG. 10. The capillary and Bond number correlation for four different combinations of exerted forces $(g, l)$. For the gravity force absent, $g=0$, a constant value of capillary number is found for larger climbing heights.

and numerical calculations are obtained in small domains in which the capillary instability may be limited geometrically.

\section{CONCLUSIONS}

The experimental and numerical studies reveal that the capillary climb opposed by gravity force starts as a single-phase flow for low climbing height, which is followed by a multiphase pattern for higher climbing heights. For the single-phase flow, a sharp interface between the liquid and gas phase exists, which transforms later into a flow front of increasing thickness as climbing progresses. The flow front thickness is a power law function of climbing time, with the power having the same value for a specific porous material regardless of the forces exerted. The power changes its value for different porous media and it decreases as permeability increases. On the other hand, for the gravity force present, distinct porous media show the same characteristic for the climb dynamics expressed as capillary versus Bond number correlation in an exponential form. In this correlation, the permeability and capillary pressure in the single-phase flow and the wetting interface between the wetted and dry fractions of porous medium are used to calculate the capillary and Bond numbers. The correlation holds regardless of multiphase flow front present for larger climbing heights. In addition to the capillary number versus the Bond number correlation, the normalized climbing height as a function of the capillary number can be used to predict the climbing dynamics. The normalized height is calculated from the characteristic heights for which the climb changes its dominant force (as a capillaryto gravity-force-dominant climb). Both exponential and power law dependences are found, where the power law applies only for the climb with the flow front developed. For the flows with the gravity force absent (driven by the capillary force only), the capillary number becomes constant after some climb height is reached. On the other hand, a very limited fingering is observed throughout the capillary climb, suggesting that the flow is stable. The stability of the flow may be caused by limited domain size (smaller than the finger size). Finally, the generality of this correlation needs to be examined further for both granular and fibrous porous media.

\section{ACKNOWLEDGMENTS}

This project was supported by the Defense Threat Reduction Agency (DTRA) and the US Army's Edgewood Chemical and Biological Center. The suggestions on the development of the technical framework from Dr. J. Savage and J. Kiple are greatly appreciated.
[1] J. Bear, Dynamics of Fluids in Porous Media (Dover Publications, Inc., New York, 1988).

[2] F. A. L. Dullien, Porous Media: Fluid Transport and Pore Structure (Academic Press, New York, 1992).

[3] H. W. Piekaar and L. A. Clarenburg, Chem. Eng. Sci. 22, 1399 (1967).

[4] I. Pezron, G. Bourgain, and D. Queret, J. Colloid Interface Sci. 173, 319 (1995).

[5] J. Ghassemzadeh, M. Hashemi, L. Sartor, and M. Sahimi, AIChE J. 47, 519 (2001).

[6] B. Markicevic and H. K. Navaz, Phys. Fluids 22, 122103 (2010).

[7] S. Supple and N. Quirke, Phys. Rev. Lett. 90, 214501 (2003).
[8] I. Battiato, P. R. Bandaru, and D. M. Tartakovsky, Phys. Rev. Lett. 105, 144504 (2010).

[9] E. W. Washburn, Phys. Rev. 17, 273 (1921).

[10] G. L. Chen and S. P. Neuman, Phys. Fluids 8, 353 (1996).

[11] D. M. Tartakovsky and C. L. Winter, SIAM J. Appl. Math. 61, 1857 (2001).

[12] A. M. Tartakovsky, S. P. Neuman, and R. J. Lenhard, Phys. Fluids 15, 3331 (2003).

[13] B. Markicevic and H. K. Navaz, Trans. Porous Media 85, 953 (2010).

[14] J. M. Bell and F. K. Cameron, J. Phys. Chem. 10, 658 (1906).

[15] W. H. Green and G. A. Ampt, J. Agric. Sci. 4, 1(1911). 
[16] R. Lucas, Kolloid-Z. 23, 15 (1918).

[17] D. A. Lockington and J. Y. Parlange, J. Colloid Interface Sci. 278, 404 (2004).

[18] R. H. Brooks and A. T. Corey, Hydrology Papers of Colorado State University 3, 1 (1964).

[19] M. A. Theodoropoulou, V. Sygouni, V. Karoutsos, and C. D. Tsakiroglou, Int. J. Multiphase Flow 31, 1155 (2005).

[20] C. D. Tsakiroglou, AIChE J. 57, 2618 (2011).

[21] T. Delker, D. B. Pengra, and P.-Z. Wong, Phys. Rev. Lett. 76, 2902 (1996).

[22] M. Lago and M. Araujo, J. Colloid Interface Sci. 234, 35 (2001).

[23] B. Bijeljic, B. Markicevic, and H. K. Navaz, Phys. Rev. E 83, 056310 (2011).

[24] P. G. Saffman and G. I. Taylor, Proc. R. Soc. London, Sect. A 245, 312 (1958).

[25] P. G. Saffman, J. Fluid Mech. 173, 73 (1986).
[26] R. Lenormand, E. Touboul, and C. Zarcone, J. Fluid Mech. 189, 165 (1988).

[27] R. Lenormand and C. Zarcone, Trans. Porous Media 4, 599 (1989).

[28] Y. C. Yortsos, B. Xu, and D. Salin, Phys. Rev. Lett. 79, 4581 (1997).

[29] Y. Meheust, G. Lovoll, K. J. Maloy, and J. Schmittbuhl, Phys. Rev. E 66, 051603 (2002).

[30] F. Plourde and M. Prat, Int. J. Heat Mass Transfer 46, 1293 (2003).

[31] G. Lovoll, Y. Meheust, K. J. Maloy, E. Aker, and J. Schmittbuhl, Energy 30, 861 (2005).

[32] D. Or, Adv. Water Resour. 31, 1129 (2008).

[33] B. Markicevic and H. K. Navaz, Int. J. Numer. Methods Heat Fluid Flow 19, 521 (2009).

[34] B. Markicevic, T. G. D'Onofrio, and H. K. Navaz, Phys. Fluids 22, 012103 (2010). 